\title{
Primary Anorectal Malignant Melanoma: Case Report and Review of Literature
}

\author{
Youcef Khenchoul $^{1}$, Abderraouf Bataiche ${ }^{2 *}$, Dounia Hellal ${ }^{3}$, Saadoune Bendjaballah ${ }^{1}$
}

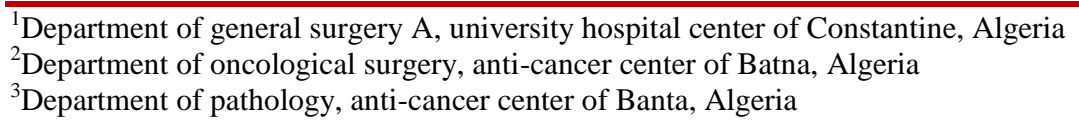

DOI: $10.36347 /$ sasjs.2022.v08i01.012

| Received: 26.11.2021 | Accepted: 01.01.2022 | Published: 30.01 .2022

*Corresponding author: Abderraouf Bataiche

Department of oncological surgery, anti-cancer center of Batna, Algeria

Abstract

Anorectal malignant melanomas are rare and aggressive tumors. The diagnosis is based on pathological exams. We reported the case of a 54 years old patient who underwent a surgical resection for rectal melanomas diagnosed after histological exams of rectal biopsy tissues.

Key words: Anorectal melanoma, epidemiology, diagnoses, therapy.

Copyright $(\mathcal{2 0 2 2}$ The Author(s): This is an open-access article distributed under the terms of the Creative Commons Attribution 4.0 International License (CC BY-NC 4.0) which permits unrestricted use, distribution, and reproduction in any medium for non-commercial use provided the original author and source are credited.

\section{INTRODUCTION}

Anorectal malignant melanomas are extremely rare and aggressive tumors representing $0.05 \%$ of all malignant colorectal diseases and 0.5 to $4.6 \%$ of all ano-rectal malignancies [1]. Diagnosis of melanoma can be straightforward based on melanin pigment, classical histomorphology, and typical immunohistochemical profiles [2]. Abdominoperineal resection was historically considered the standard of care, but multiple studies, some involving hundreds of patients, failed to report a survival benefit between APR and local excision [3].

\section{Case Report}

A 54 years old patient with remote history of diabetes type II and appendectomy, presented in emergency department for rectorrhagia for who the physical exam revealed a mass in rectal bulb which was characterized in rectal endoscopy as a $35 \mathrm{~mm}$ ulcerative hemorrhagic tumor located in the posterior wall of the rectal bulb in contact with the anorectal junction, from which biopsy was obtained. Pathological exams shows ulcerated malignant melanoma of the rectum that is comprised of spindle and epithelioid cells infiltrating the mucosa. The lesional cells contain abundant eosinophilic cytoplasm, elongated nuclei with fairly evenly dispersed chromatin, occasional intranuclear inclusions, and a brisk mitotic rate. The vascularity of the tumor is prominent. This lesion was amelanotic.
Immunostains show the lesional cells to be positive for HMB45, Melan A proteins and negative for AE1/AE3, CD56, CD45. Pelvic MRI was also performed and revealed a lower rectal mass of $34 \mathrm{~mm}$ diameter in the posterior wall with the invasion of the internal anal sphincter and the intersphincterian space. An abdominal perineal resection with anorectal amputation was carried out, along with total mesorectal excision. The patient made an uneventful recovery after surgery.

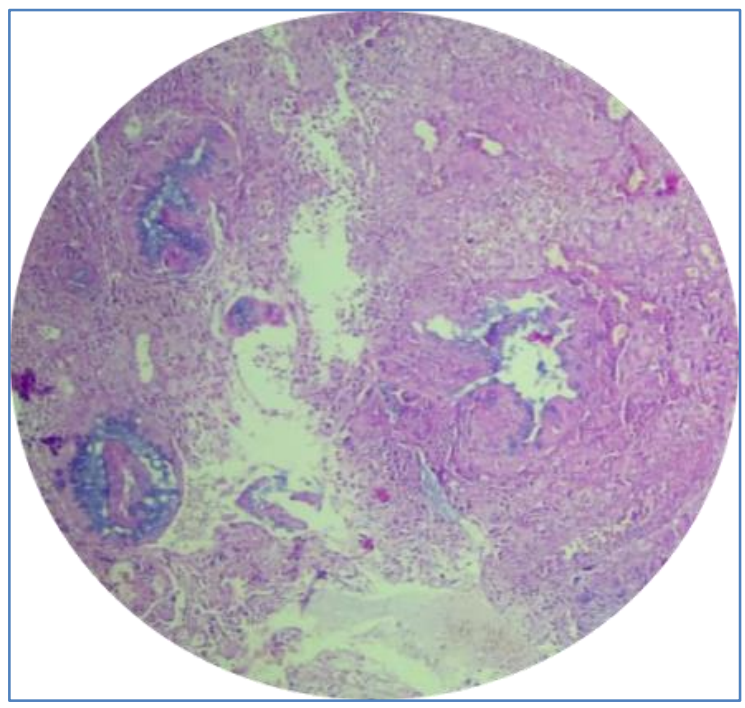

Fig-01: Low power view: ulcerating invasive melanoma 


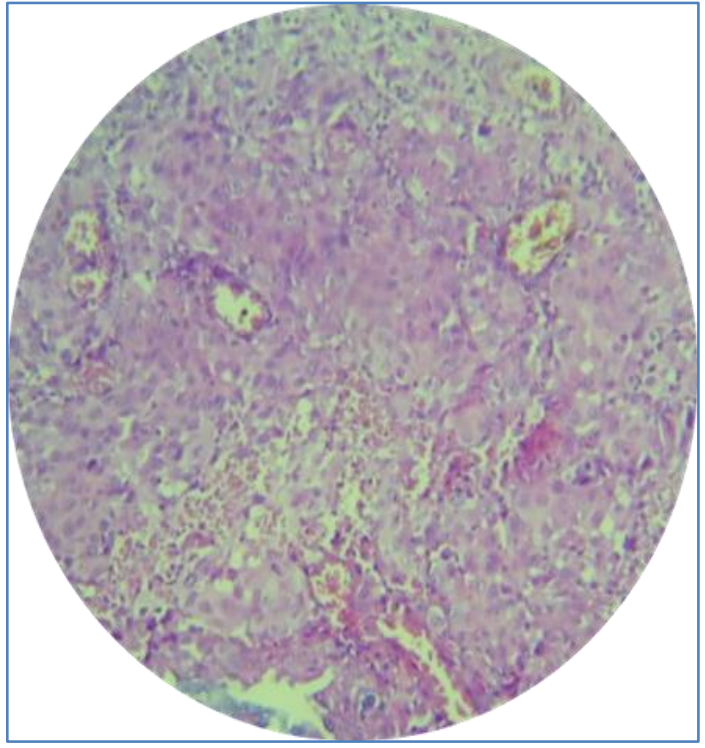

Fig-02: This high-power view of melanoma shows a neoplasm that is highly cellular, with scattered large cells, mitotic figures

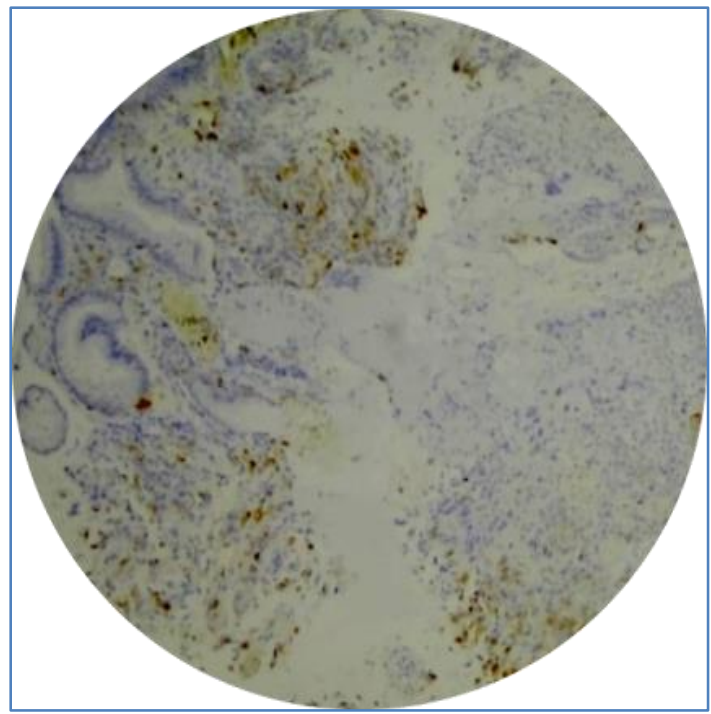

Fig-03: Melan A show a cytoplasmic staining in rectal melanoma

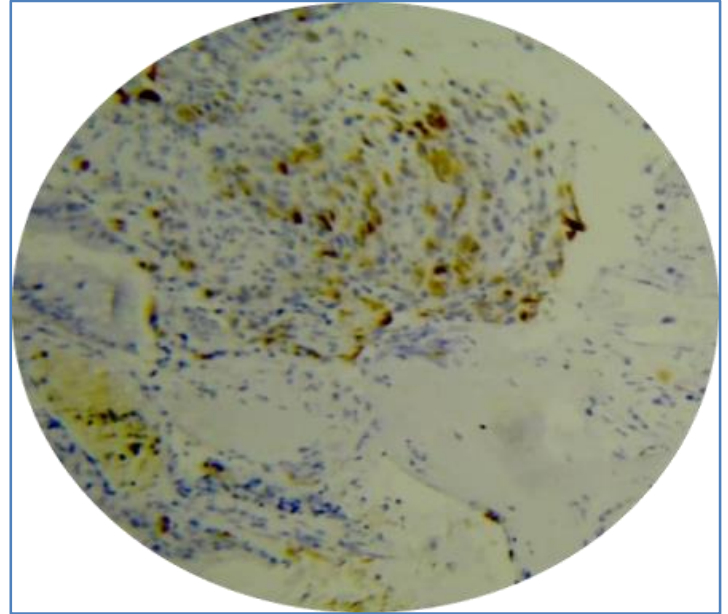

Fig-04: HMB-45 shows cytoplasmic labeling in rectal melanoma

\section{DISCUSSION}

Anorectal melanomas are rare but aggressive tumors. Compared with cutaneous melanomas, anorectal melanomas have the lowest percent of five years survivals, only $25 \%$. Best hope for survival is offered by early detection and complete surgical removal. However is usually delayed because of occult site of recurrence and unspecific symptoms diagnosis. The most common presenting symptom of anorectal melanoma is bleeding with $53-89 \%$ of patients reporting this as the predominant complaint. Other symptoms are suspicion of hemorrhoids, discomfort or pain, an anal mass, change in bowel habit, tenesmus or pruritus $[1,4,5]$. Rectorrhagia was the main symptom in our case, for which several exams were made to put in the diagnosis of rectal melanoma.

Two methods of staging in anorectal melanoma were described: 1) developed by The American Joint Commission on Cancer, which is a staging method based on depth of primary tumor, presence of tumor in lymph nodes and presence of distant metastasis (table 1). 2) Another staging system based only on disease spread: it describes local disease only as stage 1 , regional lymph node disease as stage 2 , and metastatic disease as stage $3[1,6,8]$.

Table-1: Staging classification of ARMM Stage Tumor spread

\begin{tabular}{|lc|}
\hline Stage & tumor spread \\
\hline I & Local tumor spread without infiltration of the muscular layer \\
II & Local tumor spread with infiltration of the muscular layer \\
III & Regional tumor spread and/or positive lymph node metastasis \\
IV & Disseminated tumor spread \\
\hline
\end{tabular}

Based on the American Joint Commission on Cancer classification, our tumor is classified as stage II with the infiltration of the muscular layer.

Histopathologically, AMM show considerable variability regarding the size and type of cells. They can be misdiagnosed as malignant lymphoma, small round cell sarcoma, spindle cell sarcoma, gastrointestinal stromal tumour, and epidermoid carcinoma. Thus, immunohistochemical analysis plays a pivotal role in the diagnosis of AMM. The most commonly used IHC stain in the diagnosis of AMM is Anti-S-100 protein and it is highly sensitive for melanocytic differentiation. Also, human melanoma black (HMB-45), Vimentin, 
and Melan A antibody are the melanocyte specific stains used for diagnosis of malignant melanoma [7]. In our case, diagnoses were made by imunohistochemical exam showed a positiveresults for HMB45 and Melan A proteins. Other tumors were eliminated (negative results for AE1/AE3, CD56, CD45).

There is no consensus on which surgical approach is preferred. A number of studies claim that abdominal-perineal resection (APR) is the treatment of choice because it can control better lymphatic spread and it allow to obtain larger negative margins for local control. Other studies instead have recommended only a sphincter saving excision (wide local excision) because treatment is often palliative and wide radical surgery is unnecessary mutilating. Wide local excision (WLE) is defined as a sphincter-saving operation with a defined margin around the tumor in two dimensions. The benefits of a WLE are quicker recovery, no need for a stoma, and minimal impact on bowel function [8, 10]. We adopted in our case a surgical approach, an abdominal-perineal resection was carried out with satisfying result on local control of the pathology.

No significant survival advantage was found for those treated with radiation in addition to surgery versus those managed by surgery alone. Even among patient with $\mathrm{T} 3$ or T4 disease or in those with involved lymph nodes, radiation therapy did not improve diseasefree or overall survival $[9,10]$.

\section{CONCLUSION}

Anorectal Malignant Melanoma is a rare aggressive malignancy tumor with poor outcomes and prognosis. The treatment has not been well studied and still controversial, Due to low incidence. Large-scale prospective clinical trials are needed to establish effective therapeutic approaches.

\section{REFERENCE}

1. Falch, C., Mueller, S., Kirschniak, A., Braun, M., Koenigsrainer, A., \& Klumpp, B. (2016). Anorectal malignant melanoma: curative abdominoperineal resection: patient selection with 18F-FDGPET/CT. World journal of surgical oncology, 14(1), 1-9.
2. Bell, P. D., Israel, A. K., Dunn, A. L., \& Liao, X. (2019). Primary dedifferentiated amelanotic anorectal melanoma: report of a rare case. International journal of surgical pathology, 27(8), 923-928.

3. Jensen, C., \& Kin, C. (2017). Black is the new black: Prolapsing primary anorectal melanoma. Digestive diseases and sciences, 62(11), 2991-2993.

4. Agha, R. A., Fowler, A. J., Saetta, A., Barai, I., Rajmohan, S., Orgill, D. P., \& SCARE Steering Group. (2016). A protocol for the development of reporting criteria for surgical case reports: the SCARE statement. International Journal of Surgery, 27, 187-189.

5. Agha, R. A., Fowler, A. J., Saeta, A., Barai, I., Rajmohan, S., Orgill, D. P., ... \& Rosin, D. (2016). The SCARE statement: consensus-based surgical case report guidelines. International Journal of Surgery, 34, 180-186.

6. Falch, C., Stojadinovic, A., Hann-von-Weyhern, C., Protic, M., Nissan, A., Faries, M. B., ... \& Brücher, B. L. (2013). Anorectal malignant melanoma: extensive 45-year review and proposal for a novel staging classification. Journal of the American College of Surgeons, 217(2), 324-335.

7. Xu, X., Ge, T., \& Wang, G. (2020). Primary anorectal malignant melanoma: A Case report. Medicine, 99(5).

8. Latteri, S., Teodoro, M., Malaguarnera, M., Mannino, M., Currò, G., \& La Greca, G. (2017). Abdominal perineal resection or wilde local excision in primary anorectal malignant melanoma. Case report and review. Annals of medicine and surgery, 19, 74-77.

9. Tchelebi, L., Guirguis, A., \& Ashamalla, H. (2016). Rectal melanoma: epidemiology, prognosis, and role of adjuvant radiation therapy. Journal of cancer research and clinical oncology, 142(12), 2569-2575.

10. Menon, H., Patel, R. R., Cushman, T. R., Amini, A., Seyedin, S. N., Adams, A. C., ... \& Verma, V. (2020). Management and outcomes of primary anorectal melanoma in the United States. Future oncology, 16(8), 329-338. 